\title{
Oral diseases and inflammatory burden and Alzheimer's disease among subjects aged 75 years or older
}

Antti Tiisanoja ${ }^{1}$, Anna-Maija Hannele Syrjälä ${ }^{1,2,3}$, Miia Tertsonen ${ }^{1}$, Kaija Komulainen ${ }^{4,5}$, Paula Pesonen $^{3,6}$, Matti Knuuttila ${ }^{3}$, Sirpa Hartikainen ${ }^{7,8}$, Pekka Ylöstalo ${ }^{1,3}$

Running title: Oral infections and Alzheimer's disease

Keywords: older people, Alzheimer's disease, periodontal infection, deepened periodontal pocket, dental caries, stomatitis

${ }^{1}$ Periodontology and Geriatric Dentistry, Center of Oral Health Sciences Research, University of Oulu, Oulu, Finland

${ }^{2}$ Dental Training Clinic, Social and Health services, Oulu, Finland

${ }^{3}$ Medical Research Center Oulu, Oulu University Hospital and University of Oulu, Oulu, Finland

${ }^{4}$ School of Pharmacy, University of Eastern Finland, Kuopio, Finland

${ }^{5}$ Social and Health Centre of Kuopio, Kuopio, Finland

${ }^{6}$ Faculty of Medicine, University of Oulu, Finland

${ }^{7}$ Department of Social Pharmacy, School of Pharmacy, University of Eastern Finland, Kuopio, Finland

${ }^{8}$ Unit of Clinical Pharmacology and Geriatric Pharmacotherapy, School of Pharmacy, University of Eastern Finland, Kuopio, Finland

Corresponding author:

Antti Tiisanoja

Periodontology and Geriatric Dentistry

Unit of Oral Health Sciences Research

P.O. BOX 5281

FI-90401, University of Oulu

Finland

antti.tiisanoja@oulu.fi 


\begin{abstract}
Aim: To study whether as dental caries, periodontal disease, and stomatitis, and related inflammatory burden associate with diagnosed Alzheimer's disease (AD) and dementia among older people.
\end{abstract}

Methods: The study population included 170 individuals aged $\geq 75$ years. The primary outcome was diagnosed AD and the secondary outcome any types of diagnosed dementia. Information about participants' oral diseases and related inflammatory burden were based on the clinical oral examination. Relative risks (RRs) and confidence intervals (CIs) were estimated using regression models.

Results: Dental caries, the presence of $\geq 3$ carious teeth (RR: $3.47,95 \%$ CI: $1.09-11.1$ ) and the number of carious teeth (RR: 1.24, CI: 1.11-1.39), and inflammatory burden (RR: 1.44, CI: 1.04-2.01) were associated with a higher likelihood of having AD. Also, periodontal disease and stomatitis were associated, although non-statistically, with AD and dementia. The risk estimates for any type of dementia were in most cases lower than for $\mathrm{AD}$.

Conclusion: Oral diseases and the related inflammatory burden were in most cases associated more strongly with diagnosed $\mathrm{AD}$ than dementia in general. Of the oral diseases studied, the strongest association was between dental caries and AD.

Keywords: Alzheimer's disease, dementia, oral diseases, inflammatory burden 


\section{Introduction}

Alzheimer's disease (AD) is progressive brain atrophy where an increasing accumulation of cortical senile plaques and formation of a neurofibrillary tangle, protein tau, are observed in the brain. ${ }^{1}$ Although the exact aetiology is not known, recent studies have indicated that inflammation in the central nervous system (CNS) plays a key role in the onset and progression of AD..$^{2,3}$

It has been hypothesised that oral diseases — especially inflammatory diseases — have an influence on the development or progression of $\mathrm{AD} .{ }^{4,5}$ Especially periodontitis and periapical lesions are interesting candidates, first because they cause low-grade inflammation that can spread into the brain and secondly because endotoxins of certain causative bacteria are able to penetrate blood-brain barrier. ${ }^{6,7}$ Also causative bacteria for oral diseases such as Treponema denticola, Porphyromonas gingivalis, and

Streptococcus mutans — for example — appear to have neuroinvasive properties. ${ }^{8-10}$ Information about Candida yeasts' potential effect on AD is currently miniscule, but it has been reported that Candida yeasts enhance neural inflammation ${ }^{9}$. It has been suggested that this takes place through either a systemic mycosis ${ }^{11}$ or an influx of fungal molecules into the brain. ${ }^{12}$

The aim of this cross-sectional study was to investigate whether oral diseases, measured as the number of carious teeth, the number of teeth with periodontal pockets with $\geq 4 \mathrm{~mm}$ depth, or the presence of stomatitis, are associated with diagnosed AD and dementia in general. The other aim was to study whether the inflammatory burden related to oral diseases is associated with $\mathrm{AD}$ and dementia. The hypothesis of this study was that people with dental caries, periodontal disease, or stomatitis are more likely to have AD or dementia than those who are free of these oral diseases. 


\section{Materials and methods}

\section{Study population}

The Oral Health GeMS-study is a part of the larger Geriatric Multidisciplinary Strategy for Good Care of the Elderly (GeMS) study, which was a population-based intervention study aimed at preventing disability among participants. For the GeMS study, 1000 individuals aged 75 or older were sampled from the total population living in Kuopio, in eastern Finland, on the $1^{\text {st }}$ of November in 2003. These 1000 trial subjects were divided into intervention and control groups, both consisting of 500 subjects. A Comprehensive Geriatric Assessment was carried out for 404 subjects in the intervention group. Clinical oral examination data were collected from 354 of the 404 subjects in the above-mentioned intervention group ( 27 refused and 23 died before the oral examination) in 2004 and early spring 2005. In this particular study, we further restricted the study population to nonsmoking persons with at least one natural tooth $(n=170)$.

For further information about the original GeMS-study or the Oral Health GeMS-study, we recommend articles by Karttunen et al. ${ }^{13}$ and Komulainen et al. ${ }^{14}$

\section{Comprehensive Geriatric Assessment (CGA) and interventions}

A geriatrician and a trained nurse conducted a structured clinical examination and an interview of the intervention group $(n=404)$. In the clinical examination, they assessed the participants physical and mental status and checked their medication. Laboratory tests were also taken. The questions in the interview covered the participants health, health behaviour and social life. Diagnoses were self-reported by the participants and complemented with data obtained from Special Reimbursement Registers maintained by the Social Insurance Institution of Finland, and from medical records of the Health Care Centre, home service, local hospitals and Kuopio University Hospital. If a participant 
could not answer the question, a relative and/or caregiver provided the information. The geriatrician and nurse made a home or institutional visit if a participant was unable to visit the Health Centre.

General health related interventions started in 2004 and they continued to be carried out parallel with clinical oral examinations. The interventions were aimed at preventing disability and they were given based on an individual need. These interventions included medication review and physical activity guidance. All the participants of the present study belonged to the intervention group.

\section{Outcome variables and cognitive function}

In this study, the primary outcome was diagnosed Alzheimer's disease (AD) and the secondary outcome was all diagnosed dementias, which included AD, vascular dementia, and dementia with Lewy bodies.

The diagnoses of $\mathrm{AD}$ and vascular dementia were done according to the DSM-IV criteria $^{15}$ and dementia with Lewy bodies was clinically diagnosed according to the criteria presented by McKeith et al. ${ }^{16}$ All dementia diagnoses were based on a comprehensive clinical examination carried out by a geriatrician and the diagnostic procedures included a computer tomography/magnetic resonance scan, laboratory tests and exclusion of alternative diagnoses. In addition, a history of cognitive decline was obtained by interviewing the participants and his/her relatives and examining medical records. If the participant did not meet the diagnosis criteria of $\mathrm{AD}$ or any other type dementia, they were considered as being free of these diseases.

Participants' cognitive function was assessed by using the Mini-Mental State Examination (MMSE) ${ }^{17}$ The MMSE scores ranged from 0 to 30, with higher scores indicating better cognitive function. 


\section{Clinical oral examination}

Clinical oral examination data were collected by two dentists. The examination included a structured interview of oral health and dental care habits. Before the data collection, the dentists were calibrated by examining together seven study participants for training purposes. Examinations were not repeated because of the high age of the participants and the length of the examination. All participants were examined by a dentist and a dental hygienist or dental nurse in a standardized manner based on written instructions. The clinical oral examination was conducted in a dental unit equipped with a dental chair and lamp by using a mouth mirror, WHO colour-coded periodontal probe, gauze pad, saliva suction and syringe. Most of the clinical oral examinations were conducted in the dental clinic, but if a subject preferred an examination at home, a dentist and a dental hygienist or nurse made a home or institutional visit. During a home or institutional visit, the dentist used a head lamp or flashlight if needed, and cotton rolls and gauzes were used for drying.

\section{Explanatory variables}

We used the number of teeth with periodontal pocket depth of $4 \mathrm{~mm}$ or more, the number of carious teeth, and the presence of stomatitis as explanatory variables. The periodontal pockets of all teeth were probed at two sites, mesial-buccal and distal-palatinal/lingual surfaces, but only the deepest pocket depth of each tooth was registered. In the analysis, the variable was used as: no teeth with periodontal pockets $v s$. having at least one tooth with periodontal pocket depth $\geq 4 \mathrm{~mm}$ (categorical variable). The variable was used also as a continuous explanatory variable, i.e. a number of teeth with periodontal pockets $(\geq 4$ $\mathrm{mm})$.

Dental caries was diagnosed by tactile and visual examination on five surfaces of each tooth as root caries, crown caries, crown and root caries, or carious dental radix. The tooth 
was registered as a carious tooth if any of the above-mentioned criteria was fulfilled on any surface of the tooth. Dental caries was classified into three categories: 1) no teeth with caries, 2) 1-2 teeth with caries, and 3) $\geq 3$ teeth with caries. In the analysis, the variable was used also as a continuous explanatory variable.

The oral mucosa was examined for any oral lesions on the buccal tissues, palate, tongue, under the tongue, gingiva, and alveolar ridges. All findings were registered by their location, colour, and surface structure, but not the size of the lesion. The presence of stomatitis was determined by visual inspection of any smooth or nodular redness in the oral mucosa. Denture stomatitis was determined if similar lesions were visible under removable dentures. For this study, stomatitis and denture stomatitis were combined and in the analyses stomatitis was used as dichotomous variable (No vs. Yes).

Inflammatory burden was determined by giving numeric values to each of the oral diseases according to their severity and then adding up the values. Periodontal disease was classified into three point-categories $\left(\mathrm{N}_{\text {periodontal }}\right)$ : 0 points $=$ no periodontal pockets, 1 point $=$ one to three periodontal pockets $(\geq 4 \mathrm{~mm})$, and 2 points $=$ four or more periodontal pockets $(\geq 4 \mathrm{~mm})$. Dental caries was also classified into three categories $\left(\mathrm{N}_{\text {caries }}\right): 0$ points $=$ no dental caries, 1 point $=$ one to two carious teeth, and 2 points $=$ three or more carious teeth. Stomatitis was classified into two point-categories ( $\left.\mathrm{N}_{\text {stomatitis }}\right)$ : 0 points $=$ no stomatitis, 1 point $=$ stomatitis

$$
\text { Inflammatory burden }=\Sigma\left(\mathrm{N}_{\text {periodontal }}+\mathrm{N}_{\text {caries }}+\mathrm{N}_{\text {stomatitis }}\right)
$$

A higher number represents a more severe burden caused by the inflammatory oral diseases and the maximum value is five.

Potential confounding factors 
Education was classified into two categories based on the number of years of formal education: a lower education level being compulsory comprehensive school or less $(<7$ years), a higher education level being secondary school or occupational education $(\geq 7$ years).

Participants diagnosed diseases were identified by combining data from the interview, medical records, and medication registers. The following diagnosed diseases were included: diabetes, hypertension, coronary heart disease, and stroke.

Dementia among relatives was assessed during the interview with a question: Who of your close relatives has dementia? Participants reported close relatives such as grandparents, parents, siblings, or uncles/aunts having a dementia or suspicion of dementia.

\section{The statistical methods}

Poisson regression models with robust error variance ${ }^{18}$ were used to estimate relative risk (RR) with a 95\% confidence interval (CI 95\%). All the models were adjusted for age, gender, education, diabetes, hypertension, coronary heart disease, stroke, and dementia among relatives. SPSS (Chicago Ill., USA) 24.0 software for Windows was used to analyse the data.

\section{Results}

The characteristics of the study population according to cognitive status are shown in Table 1. Of the total population, 11\% $(n=18)$ had AD and four percent $(n=7)$ had other form of dementia. The MMSE mean scores were 28 in cognitively healthy and 18 in participants with any form of dementia. The proportion of AD among people with dental caries was $13 \%$, among persons with periodontal pockets $(\geq 4 \mathrm{~mm}), 11 \%$, and among people with stomatitis, $14 \%$. 
Unadjusted relative risks for explanatory variables are presented in Table 2. Adjusted relative risks from regression models are presented in Table 3. After adjustment for risks for dementia, it was found that persons with $\geq 3$ carious teeth had a higher likelihood of having AD when compared to those with no carious teeth (RR: 3.47, CI: 1.09-11.1). For the continuous variable (the number of carious teeth) the RR was $1.24, \mathrm{CI}: 1.11-1.39$ (Table 3).

Persons with deepened periodontal pocket with pocket depth $\geq 4 \mathrm{~mm}$ had an increased, although not statistically significant, likelihood of having AD when compared to persons without periodontal pockets with pocket depth $\geq 4 \mathrm{~mm}$ (RR: $1.54, \mathrm{CI}: 0.52-4.56)$. For the continuous variable, the number of teeth with deepened periodontal pocket with pocket depth $\geq 4 \mathrm{~mm}$, the RR was 1.03, CI: 0.96-1.10 (Table 3). For stomatitis the RR was 1.17 (CI: 0.36-3.79) and for the continuous inflammatory burden variable, 1.44 (CI: 1.042.01).

In addition to $\mathrm{AD}$, an association with having any type of dementia in general was also studied. Regarding periodontitis, stomatitis, and inflammatory burden, the analyses showed in most cases lower risk estimates for diagnosed dementia compared to the risk estimates of $\mathrm{AD}$ (Table 3 ).

\section{Discussion}

We found dental caries, periodontal disease, stomatitis, and inflammatory burden to be associated with diagnosed dementia and AD. The results also showed that periodontal disease, stomatitis, and inflammatory burden associated with diagnosed AD more strongly than with diagnosed dementia in general. However, it must be pointed out that due to the low number of $\mathrm{AD}$ cases and the relatively small data set, most of the risk estimates were statistically non-significant. 
Due to the complexity of AD's pathology and the multiple risk factors (sociodemographic, general health etc.) associated with $\mathrm{AD}$, it is challenging to determine how oral diseases have effect on AD. According to the current understanding of AD's pathology an inflammation is an important component in the onset and progression of the disease. ${ }^{2,19}$ It has been hypothesized that certain oral diseases could have an influence on $\mathrm{AD}$ either by causing a low-grade inflammation, ${ }^{7,20}$ by activating microglia cells via proinflammatory molecules, ${ }^{21}$ or by microbial invasion into the brain. ${ }^{8,9,10,22,23}$ It has also been speculated that the effects of oral diseases on cognitive function might be mediated through changes in diet that in turn are related to impaired mastication. ${ }^{24,25}$

It must be emphasized that this study has a cross-sectional design and that we cannot draw any conclusions about the causal role of oral diseases in the development and progression of $\mathrm{AD}$ because we do not have data about the initiation and progression of $\mathrm{AD}$ or other dementias or oral diseases. This prevents us from determining whether oral diseases are a cause or a consequence of dementia. This problem has also been acknowledged in a recent systematic review focusing on longitudinal studies. ${ }^{26}$

Since in many cases oral diseases are a consequence of dementia, it is by no means unexpected that several previous studies have reported an association between oral diseases and dementia or cognitive decline. Regarding dental caries, it is important to note that there are findings for ${ }^{27}$ and against. ${ }^{28,29}$ The findings of the present study related to periodontal condition and $\mathrm{AD}$ are — to some extent—in line with a number of earlier studies, that have shown gingival inflammation ${ }^{30}$, alveolar bone loss, or probing depth to be associated with low cognition scores. ${ }^{27,31}$ Also, the finding related to stomatitis is in line with earlier studies that have shown an association between fungal infections and AD. ${ }^{11,12,32}$ Based on these findings, it is not surprising that also inflammatory burden, originally resulting from dysbiotic oral microbiome, ${ }^{33,34}$ was associated with AD. 


\section{Strengths and limitations}

In this study, diagnosed $\mathrm{AD}$ was used as a primary outcome and any type of diagnosed dementias in general was used a secondary outcome. The use of two outcomes made it possible for us to study the possible specificity of the associations.

Diagnoses of Alzheimer's disease and other dementias were determined by a physician with the help of the Diagnostic and Statistical Manual of Mental Disorder, fourth edition. ${ }^{15}$ The participants without any dementia diagnose were considered as non-cases despite the fact that some of them had a mild cognitive impairment. This may have caused the attenuation of risk estimates. When studying $\mathrm{AD}$, the risk estimates may have been further attenuated due to the fact that the participants with other types of dementias than $\mathrm{AD}$ were considered as non-cases.

In this study, periodontal condition was assessed by probing the depths of periodontal pockets and recorded as the number of teeth with periodontal pocket depth $\geq 4 \mathrm{~mm}$. This measurement was robust, as only the deepest periodontal pocket of each tooth was registered. A limitation in the assessment of periodontal condition was that clinical attachment loss was not measured. This recording method might have underestimated the extent and severity of periodontal disease.

Classification of dental caries was based on a restorative treatment need, measured by a visual and tactile examination of each surface of the tooth and registered at tooth level. This robust classification method was used because of the time limitation and because the Oral Health GeMS focused on individual treatment need. A limitation related to dental caries detection was that radiographs were not taken routinely, most likely leading to underregistration of dental caries. Due to the absence of periapical radiographs, we cannot even speculate on the presence of periapical lesions. 
The current study differs from the previous ones by the inclusion of different oral diseases as explanatory variables. Most of the earlier studies have focused on only one oral disease at a time, such as periodontitis, dental caries or stomatitis, whereas this study included all the above-mentioned diseases. In addition, a specific variable was used to measure the inflammatory burden. Although, this variable takes into account the severity of dental caries and periodontitis, it is still important to keep in mind that this variable is a fairly crude measure. At the moment there is no clear "gold standard" for measuring inflammatory burden, and different methods have been used as a measure: based on serological tests ${ }^{35}$, questionnaires on past exposure to infectious diseases ${ }^{36}$, or clinical oral parameters.

It is known that dementia risk is associated with traditional risk factors of cardiovascular diseases, such as high blood pressure, smoking, diabetes, and high cholesterol. ${ }^{37}$ Thus, we applied multivariate regression models to take into account the confounding effect of other competing risks. The potential confounding effect of smoking was controlled by excluding smokers from the study. Despite our efforts, it is possible that residual confounding may exist, for example related to attitudinal factors, like attitudes towards physical activity, exercise and diet.

\section{Implications}

According to current knowledge, systemic inflammation can become several-fold in the brain, leading to an accumulation of proteins. This accumulation of proteins reduces the function of neurons and eventually destroys them. Although there is no conclusive or strong evidence of the role of oral diseases on cognitive brain functions, from the point of view of general health it is important to reduce oral derived systemic infection and inflammation. By enhancing oral hygiene and treating oral diseases in patients of all ages 
it is possible to improve oral health and thus, reduce consequent low-grade systemic inflammation and prevent transient bacteraemia.

\section{Conclusion}

Within the limitations of this study, we conclude that oral diseases and their inflammatory burden were in most cases associated more specifically with diagnosed Alzheimer's disease than diagnosed dementias in general. Of the oral diseases studied, dental caries was found to be associated most strongly with diagnosed Alzheimer's disease and dementia. 


\section{REFERENCES:}

1. Miklossy J. Alzheimer's disease - a neurospirochetosis. Analysis of the evidence following Koch's and Hill's criteria. J Neuroinflammation 2011; 8: 90 doi: 10.1186/1742-2094-8-90.

2. Heneka MT, O'Banion MK, Terwel D, Kummer MP. Neuroinflammatory processes in Alzheimer's disease. J Neural Transm 2010; 117: 919-947.

3. Holmes C, Cunningham C, Zotova E, et al. Systemic inflammation and disease progression in Alzheimer disease. Neurology 2009; 73: 768-774.

4. Noble JM, Scarmeas N, Papapanou PN. Poor oral health as a chronic, potentially modifiable dementia risk factor: review of the literature. Curr Neurol Neurosci Rep 2013; 13: 384. doi: 10.1007/s11910-013-0384-x.

5. Teixeira FB, Saito MT, Matheus FC, et al. Periodontitis and Alzheimer's Disease: A Possible Comorbidity between Oral Chronic Inflammatory Condition and Neuroinflammation. Front Aging Neurosci 2017; 9: 327. doi:10.3389/fnagi.2017.00327.

6. Scannapieco F, Cantos A. Oral inflammation and infection, and chronic medical diseases: implications for the elderly. Periodontolgy 2000 2016; 72: 153-175.

7. Gomes M, Blattner T, Sant'Ana Filho M, et al. Can apical periodontitis modify systemic levels of inflammatory markers? A systematic review and metaanalysis. J Endod 2013; 39: 1205-1217.

8. Poole S, Singhrao SK, Kesavalu L, Curtis MA, Crean S. Determining the presence of periodontopathic virulence factors in short-term postmortem Alzheimer's disease brain tissue. J Alzheimers Dis 2013; 36: 665-677.

9. Olsen I, Singhrao SK. Can oral infection be a risk factor for Alzheimer's disease? J Oral Microbiol 2015; 7: 29143. doi:10.3402/jom.v7.29143.

10. Tonomura $\mathrm{S}$, Ihara $\mathrm{M}$, Kawano $\mathrm{T}$ et al. Intracerebral hemorrhage and deep microbleeds associated with cnm-positive Streptococcus mutans; a hospital cohort study. Sci Rep 2016; 6: 20074. doi:10.1038/srep20074.

11. Alonso R, Pisa D, Rábano A, Carrasco L. Alzheimer's disease and disseminated mycoses. Eur J Clin Microbiol Infect Dis 2014; 33:1125-1132.

12. Castellani RJ, Perry G, Smith MA. The role of novel chitin-like polysaccharides in Alzheimer disease. Neurotox Res 2007; 12: 269-274.

13. Karttunen NM, Turunen J, Ahonen R, Hartikainen S. More attention to pain management in community-dwelling older persons with chronic musculoskeletal pain. Age Ageing 2014; 43: 845-850.

14. Komulainen K, Ylöstalo P, Syrjälä AM, et al. Determinants for preventive oral health care need among community-dwelling older people: a population-based study. Spec Care Dentist 2014; 34:19-26.

15. Tucker G, Popkin M, Caine E, et al. Delirium, dementias, and Amnestic and Other Cognitive Disorders. In: Diagnostic and statistical manual of mental 
disorders: DSM-IV. $4^{\text {th }}$ ed. Washington, D.C: American Psychiatric Association. $1994 ; 123-163$.

16. McKeith IG, Galasko D, Kosaka K et al. Consensus guidelines for the clinical and pathologic diagnosis of dementia with Lewy bodies (DLB): report of the consortium on DLB international workshop. Neurology 1996; 47: 1113-1124.

17. Folstein MF, Folstein SE, McHugh PR. 'Mini-mental state'. A practical method for grading the cognitive state of patients for the clinician. J Psychiatr Res 1975; 12: 189-198.

18. Zou G. A modified Poisson Regression approach to prospective studies with binary data. Am J Epidemiol 2004; 159: 702-706.

19. Liu C, Cui G, Zhu M, Kang X, Guo H. Neuroinflammation in Alzheimer's disease: chemokines produced by astrocytes and chemokine receptors. Int J Clin Exp Pathol 2014; 7: 8342-8355.

20. Kamer AR, Craig RG, Dasanayake AP, Brys M, Glodzik-Sobanska L, de Leon MJ. Inflammation and Alzheimer's disease: possible role of periodontal diseases. Alzheimer's dement 2008; 4: 242-250.

21. Sochocka M, Zwolińska K, Leszek J. The Infectious Etiology of Alzheimer's Disease. Curr Neuropharmacol 2017; 15: 996-1009.

22. Pisa D, Alonso R, Rábano A, Rodal I, Carrasco L. Different brain regions are infected with fungi in Alzheimer's disease. Sci Rep 2015; 5: 15015. doi:10.1038/srep15015.

23. Riviere GR, Riviere KH, Smith KS. Molecular and immunological evidence of oral Treponema in the human brain and their association with Alzheimer's disease. Oral Microbiol Immunol 2002; 17: 113-118.

24. Kim JM, Stewart R, Prince M, Kim SW, Yang SJ, Shin IS, Yoon JS. Dental health, nutritional status and recent-onset dementia in a Korean community population. Int J Geriatr Psychiatry 2007; 22: 850-855.

25. Oh B, Han DH, Han KT, Liu X, Ukken J, Chang C, Dounis K, Yoo JW. Association between residual teeth number in later life and incidence of dementia: A systematic review and meta-analysis. BMC Geriatr 2018; 18: 48. doi: 10.1186/s12877-018-0729-z.

26. Wu B, Fillenbaum GG, Plassman BL, Guo L. Association between oral health and cognitive status: a systematic review. J Am Geriatr Soc 2016; 64: 739-751.

27. Kaye EK, Valencia A, Baba N, Spiro A 3rd, Dietrich T, Garcia RI. Tooth loss and periodontal disease predict poor cognitive function in older men. $J \mathrm{Am}$ Geriatr Soc 2010; 58: 713-718.

28. Chalmers JM, Carter KD, Spencer AJ. Caries incidence and increments in community-living older adults with and without dementia. Gerodontology 2002; 19: 80-94. 
29. Ellefsen B, Holm-Pedersen P, Morse DE, et al. Assessing caries increments in elderly patients with and without dementia: A one-year follow-up study. $J \mathrm{Am}$ Dent Assoc 2009; 140: 1392-1400.

30. Stewart R, Weyant RJ, Garcia ME, et al. Adverse oral health and cognitive decline: the health, aging and body composition study. J Am Geriatr Soc 2013; 61: $177-184$.

31. Batty GD, Li Q, Huxley R, et al. Oral disease in relation to future risk of dementia and cognitive decline: prospective cohort study based on the Action in Diabetes and Vascular Disease: Preterax and Diamicron Modified-Release Controlled Evaluation (ADVANCE) trial. Eur Psychiatry 2013; 28:49-52.

32. Hoffmann M, Muniz J, Carroll E, De Villasante J. Cryptococcal meningitis misdiagnosed as Alzheimer's disease: complete neurological and cognitive recovery with treatment. J Alzheimers Dis 2009; 16: 517-520.

33. Deng ZL, Szafrański SP, Jarek M, Bhuju S, Wagner-Döbler I. Dysbiosis in chronic periodontitis: Key microbial players and interactions with the human host. Sci Rep 2017; 16: 3703. doi: 10.1038/s41598-017-03804-8.

34. Tanner ACR, Kressirer CA, Rothmiller S, Johansson I, Chalmers NI. The Caries Microbiome: Implications for Reversing Dysbiosis. Adv Dent Res 2018; 29: 7885 .

35. Elkind MSV. Infectious burden: a new risk factor and treatment target for atherosclerosis. Infect Disord Drug Targets. 2010; 10: 84-90.

36. Grau AJ, Preusch MR, Palm F, Lichy C, Becher H, Buggle F. Association of symptoms of chronic bronchitis and frequent flu-like illnesses with stroke. Stroke 2009; 40: 3206-3210.

37. Sindi S, Mangialasche F, Kivipelto M. Advances in the prevention of Alzheimer's Disease. F1000Prime Rep 2015; 7:50. doi:10.12703/P7-50. 
Table 1. Basic characteristic of the study population

\begin{tabular}{|c|c|c|c|}
\hline Variable & $\begin{array}{l}\text { Total study } \\
\text { population } \\
\mathrm{n}=170\end{array}$ & $\begin{array}{l}\text { Participants with } \\
\text { Alzheimer's } \\
\text { disease } \\
\mathrm{n}=18 \\
\end{array}$ & $\begin{array}{l}\text { Participants } \\
\text { with dementia } \\
\quad n=25\end{array}$ \\
\hline \multicolumn{4}{|l|}{$\begin{array}{l}\text { Sociodemographic } \\
\text { variables }\end{array}$} \\
\hline Mean age $( \pm S D)$ & $80.9(4.04)$ & $82.2(3.86)$ & $82.5(3.73)$ \\
\hline $\begin{array}{l}\text { Gender, proportion of males, } \\
\%\end{array}$ & 30 & 22 & 28 \\
\hline Education $>7$ years, $\%$ & 54 & 39 & 40 \\
\hline Institutionalized, \% & 7 & 28 & 28 \\
\hline \multicolumn{4}{|l|}{ Dental variables } \\
\hline $\begin{array}{l}\text { Number of teeth } \\
\text { mean }( \pm S D)\end{array}$ & $14.1(8.20)$ & $10.9(6.96)$ & $9.4(6.79)$ \\
\hline $\begin{array}{l}\text { Number of carious teeth, } \\
\text { mean }( \pm S D)\end{array}$ & $1.35(2.23)$ & $3.61(4.35)$ & $2.88(3.93)$ \\
\hline \multicolumn{4}{|l|}{ Number of carious teeth, $\%$} \\
\hline 0 & 50 & 39 & 44 \\
\hline $1-2$ teeth with caries & 31 & 11 & 16 \\
\hline$\geq 3$ teeth with caries & 19 & 50 & 40 \\
\hline $\begin{array}{l}\text { Number of teeth with } \\
\text { periodontal pocket depth } \geq 4 \\
\text { mm, mean }( \pm \text { SD) }\end{array}$ & $2.54(3.67)$ & $2.22(3.25)$ & $2.32(3.17)$ \\
\hline \multicolumn{4}{|l|}{ Periodontal condition, $\%$} \\
\hline $\begin{array}{l}\text { No teeth with periodontal } \\
\text { pockets }\end{array}$ & 44 & 44 & 44 \\
\hline $\begin{array}{l}\geq 1 \text { tooth with periodontal } \\
\text { pocket depth } \geq 4 \mathrm{~mm}\end{array}$ & 56 & 56 & 56 \\
\hline Stomatitis, \% & 12 & 17 & 12 \\
\hline
\end{tabular}


Inflammatory burden, mean $( \pm \mathrm{SD})$

\section{General health-related}

\section{variables}

Alzheimer's disease, \%

Cognitively intact, \%

Dementia among relatives, $\%$

MMSE, mean $( \pm$ SD)

Stroke, \%

Hypertonia, \%

Diabetes, $\%$

Coronary heart disease, $\%$

Number of drugs, mean $( \pm$ $\mathrm{SD})$
11

100

72

85

0

0

8

17

12

$26.1(5.58)$

$18.2(6.96)$

$18.2(6.71)$

7

0

4

47

28

28

11

0

4

37

28

32

$6.1(3.78)$

$5.61(3.26)$

$6.44(3.71)$

SD, Standard deviation; MMSE, Mini-Mental State Examination. 
Table 2. Relation between study variables and Alzheimer's disease and dementia in general

Variable

Alzheimer's disease vs. All diseases causing other participants dementia vs.

RR (CI 95\%) cognitively healthy RR (CI 95\%)

\section{Sociodemographic variables}

\begin{tabular}{|c|c|c|}
\hline Age (continuous) & $1.07(0.99-1.15)$ & $1.08(1.02-1.15)$ \\
\hline \multicolumn{3}{|l|}{ Gender } \\
\hline Male & 1.0 & 1.0 \\
\hline Female & $1.50(0.52-4.34)$ & $1.10(0.49-2.48)$ \\
\hline \multicolumn{3}{|l|}{ Education } \\
\hline$\geq 7$ years & 1.0 & 1.0 \\
\hline$<7$ years & $1.47(0.56-3.85)$ & $1.28(0.56-2.91)$ \\
\hline \multicolumn{3}{|l|}{ Dental variables } \\
\hline Number of teeth (continuous) & $0.95(0.91-1.00)$ & $0.93(0.88-0.97)$ \\
\hline Dental caries (continuous) & $1.23(1.15-1.32)$ & $1.18(1.11-1.26)$ \\
\hline \multicolumn{3}{|l|}{ Number of carious teeth } \\
\hline 0 teeth & 1.0 & 1.0 \\
\hline $1-2$ teeth & $0.46(0.10-2.12)$ & $0.58(0.20-1.74)$ \\
\hline$\geq 3$ teeth & $3.42(1.39-8.40)$ & $2.42(1.14-5.13)$ \\
\hline $\begin{array}{l}\text { Number of teeth with periodontal } \\
\text { pocket depth } \geq 4 \mathrm{~mm}\end{array}$ & $0.97(0.86-1.11)$ & $0.98(0.89-1.09)$ \\
\hline \multicolumn{3}{|l|}{ Periodontal condition } \\
\hline No teeth with periodontal pockets & 1.0 & 1.0 \\
\hline $\begin{array}{l}\geq 1 \text { tooth with periodontal pocket } \\
\text { depth } \geq 4 \mathrm{~mm}\end{array}$ & $0.99(0.41-2.38)$ & $1.01(0.49-2.08)$ \\
\hline \multicolumn{3}{|l|}{ Stomatitis } \\
\hline No & 1.0 & 1.0 \\
\hline Yes & $1.42(0.45-4.49)$ & $0.97(0.32-2.96)$ \\
\hline Inflammatory burden & $1.31(0.95-1.81)$ & $1.18(0.90-1.54)$ \\
\hline \multicolumn{3}{|l|}{ General health related variables: } \\
\hline \multicolumn{3}{|l|}{ Dementia among relatives } \\
\hline No & 1.0 & 1.0 \\
\hline Yes & $3.44(1.06-11.1)$ & $2.38(0.78-7.28)$ \\
\hline \multicolumn{3}{|l|}{ Stroke } \\
\hline No & 1.0 & 1.0 \\
\hline Yes & $\dagger$ & $0.62(0.09-4.18)$ \\
\hline \multicolumn{3}{|l|}{ Hypertension } \\
\hline No & 1.0 & 1.0 \\
\hline Yes, or formerly & $0.44(0.16-1.20)$ & $0.47(0.20-1.07)$ \\
\hline \multicolumn{3}{|l|}{ Diabetes } \\
\hline No & 1.0 & 1.0 \\
\hline Yes & $\dagger$ & $0.35(0.05-2.45)$ \\
\hline Number of drugs (continuous) & $0.96(0.86-1.08)$ & $1.02(0.94-1.11)$ \\
\hline
\end{tabular}

$\mathrm{RR}$, relative risk; CI, confidence interval, $\uparrow$ not able to calculate risk estimates. 
Table 3. Relation of study variables to Alzheimer's disease and dementia in general. Results of multivariate regression models

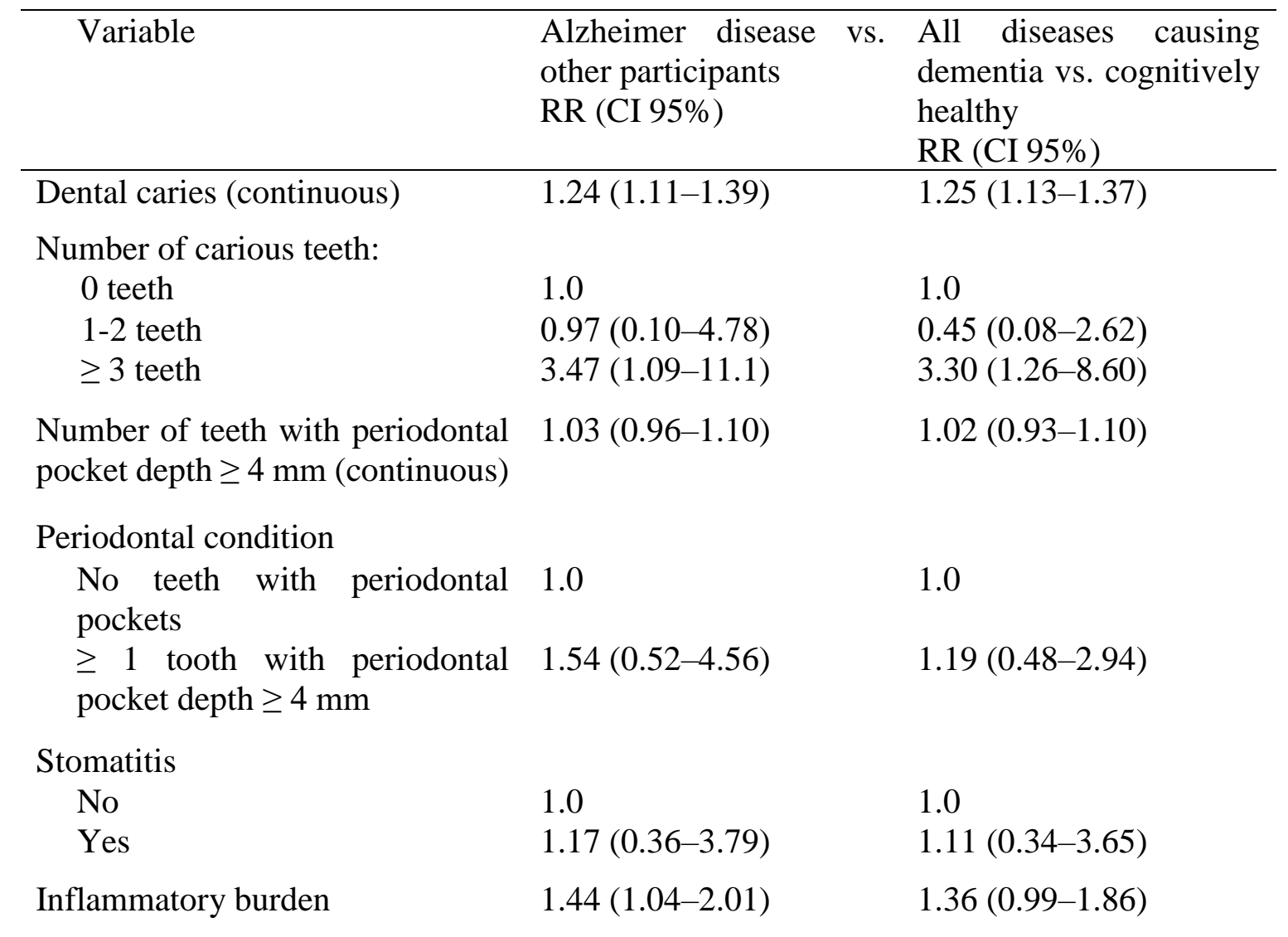

RR, relative risk; CI, confidence interval. Models were adjusted for age, gender, education, diabetes, hypertension, coronary heart disease, stroke, and dementia among relatives. 